\title{
Converging Evolutionary, Environmental and Clinical Ideas on Folate Metabolism
}

\author{
Patrice Jones $^{1}$, Emma Beckett ${ }^{1}$, Zoe Yates $^{1}$, Martin Veysey ${ }^{2}$ and Mark Lucock ${ }^{1 *}$ \\ ${ }^{1}$ University of Newcastle, Ourimbah, New South Wales, Australia; ${ }^{2}$ Central Coast Local Health District, Gosford, New South Wales, \\ Australia
}

\begin{abstract}
Background \& objectives: Folate is a UV-labile vitamin, critical to maintenance of DNA synthesis and repair mechanisms. Evidence supports a number of folate-dependent enzymes as being UV-responsive, suggesting that the activity of pathways operating through such enzymes may differ between geographic areas of varied UV exposure. Therefore, we explored the possible association of global UV levels with the prevalence of common polymorphisms in folate-metabolism enzymes. Methods: Data from the PubMed electronic database was collated for the polymorphisms MTHFR-C677T, MTHFR-A1298C, cSHMT-C1420T, TYMS 28bp 2R $>3 \mathrm{R}$, TYMS 3'UTR ins/del and DHFR $19 \mathrm{bp}$ deletion. Data extracted were analysed by the latitude of the study locations, as a surrogate measure of population UV exposure. Results: Frequency of the MTHFR-C677T and MTHFR-A1298C polymorphisms was positively associated with latitude, while a negative association was observed between latitude and frequency of the $C S H M T$ C1420T and TYMS 28bp 2R>3R variants. Conclusions: These findings provide novel evidence suggestive of folate genotypes being selected to maintain homeostasis between folate-dependent de novo thymidylate synthesis and methylation pathways in environments of differing UV levels. To the authors' knowledge, this is the first study to report significant associations between latitude and the occurrence of MTHFR-A1298C, TYMS 28bp 2R>3R and cSHMT-C1420T polymorphisms. On-going studies are required to further explore the biological significance of these findings.
\end{abstract}

\section{Introduction}

Evolution by natural selection is the principle mechanism of adaptive biological change, the simplest yet most important theory in the life sciences. The role of evolution is central to health and disease, with the application of evolution to medicine referred to

Keywords: Folate, DNA synthesis; DNA repair; Ultraviolet radiation; Polymorphism. Abbreviations: 5,10-methyleneTHF, 5,10-methylenetetrahydrofolate; THF, tetrahydrofolate; DHF, dihydrofolate; dUMP, deoxyuridine monophosphate; dTMP, deoxythymine monophosphate; 5-methylTHF, 5-methyltetrahydrofolate; cSHMT, cytosolic serinehydroxymethyl transferase; mSHMT, mitochondrial serinehydroxymethyl transferase; MTHFR, methylenetetrahydrofolate reductase; TYMS, thymidylate synthase; DHFR, dihydrofolate reductase.

Received: 01 June 2016; Revised: 01 August 2016; Accepted: 16 August 2016

DOI: 10.14218/ERHM.2016.00003b

*Correspondence to: Mark Lucock, Human Molecular Nutrition Laboratory, School of Environmental \& Life Sciences, University of Newcastle, Brush Rd, Ourimbah NSW 2258, Australia. Tel: +61-2-4348-4109, Fax: +61-2-4348-4145, E-mail: mark. lucock@newcastle.edu.au as evolutionary medicine or Darwinian medicine. Evolutionary medicine complements traditional science-based medicine, and is an approach that highlights humankind's slow adaptation to a rapidly changing environment, and which examines our continuing susceptibility to disease within the context of continuing evolution. This approach has utility in exploring the 'hows' and 'whys' of biology, exploring not only the cause of disease, but reasons for its long-term occurrence. ${ }^{1}$ The need for an approach offering a more holistic and comprehensive view of the body and disease is highlighted in the quote by evolutionary biologist Theodosius Dobzhansky: "Nothing in biology makes sense, except in the light of evolution". 2 The usefulness of applying evolutionary principles to medicine becomes apparent when we view the human body as what it truly is, a product of natural selection, with a particular focus on our reproduction, and not our health or well-being, being favoured by evolution.

Passing on genetic material is the prime objective of any living species, with the maintenance of this material's stability being fundamental to the maintenance of life. A primary dichotomy that challenges this objective is the fact that DNA, the carrier of our genetic information, is a chemical entity that is under constant assault by endogenous and environmental agents. Arguably the most pervasive environmental agent that attacks DNA is solar ultraviolet radiation (UVR), a potent genotoxic agent capable of inducing DNA damage and decreasing genomic stability. 3,4 The effect of UVR on DNA is accredited to the DNA nucleobases of irradiated cells absorbing photons in the UV region, resulting in several cytosolic effects, mutagenic DNA lesions and DNA strand breaks. ${ }^{3}$ To counteract such biological effects, organisms such as humans have evolved a number of cellular repair mechanisms.

The study of DNA damage and repair pathways is clearly important when considering the biological relevance of these pathways in disease processes. DNA damage-response pathways are some of most important in cancer development, with genomic instability being a key feature of these diseases. ${ }^{5,6}$ DNA damage is also linked to neurodegenerative disorders, with the accumulation of DNA lesions in neurons being associated with Alzheimer's, Huntington's and Parkinson's diseases. ${ }^{7,8}$ Alterations in DNA damage-response pathways have been further associated with immune deficiency, infertility, premature aging and the development of atherosclerosis. ${ }^{9-11}$

Folate, a water-soluble B-vitamin, plays an essential role in DNA stability and repair due to its actions as a cofactor for folatedependent enzymes involved in nucleotide synthesis and DNA methylation. ${ }^{12}$ Therefore, since folate status modulates both genetic stability and DNA methylation, these critical indices are determined by both dietary intake and the activity of a number of folate metabolism enzymes, many of which exist in polymorphic form. ${ }^{13}$

Numerous forms of natural folates may exist in the cell. ${ }^{14}$ 


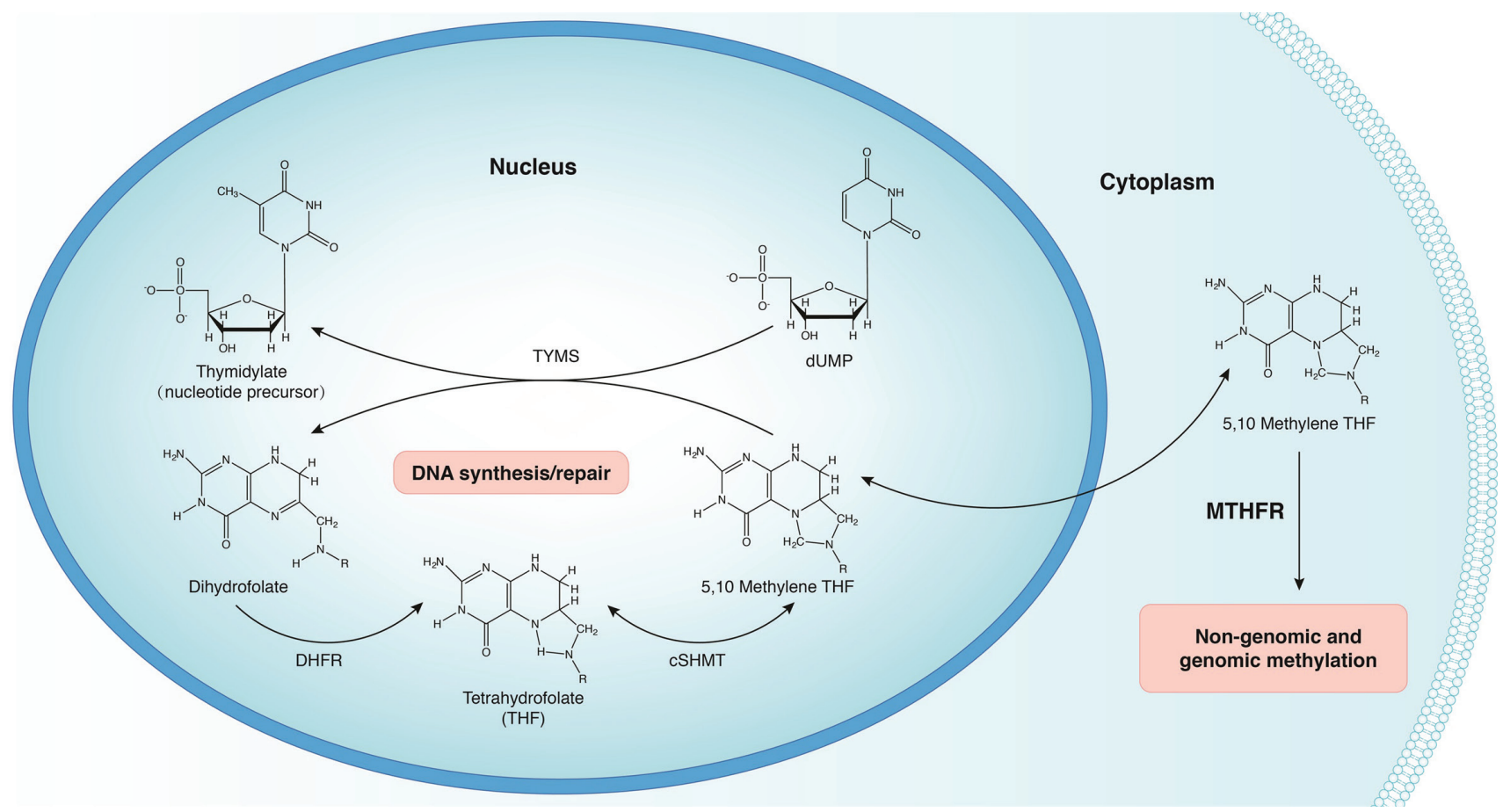

Fig. 1. 5,10-methyleneTHF synthesis and utilization in the cell. 5,10-methyleneTHF acts as the cofactor in both the de novo thymidylate and homocysteine remethylation pathways in the nucleus and cytoplasm respectively. First generated by the action of cSHMT, this vitamer can be used in the TYMS-catalysed conversion of dUMP to thymidylate, generating DHF. DHFR then catalyses the reduction of DHF back to THF in order to fuel further cycles of thymidylate synthesis. Alternatively, this substrate may be redirected toward the remethylation process catalysed by the MTHFR enzyme. ${ }^{14-16}$

Folate, as 5,10- methylenetetrahydrofolate (5,10-methyleneTHF) acts as the cofactor in both the de novo thymidylate and methionine pathways. The enzymes that constitute the de novo thymidylate pathway include cytosolic serinehydroxymethyl transferase (cSHMT), thymidylate synthase (TYMS) and dihydrofolate reductase (DHFR). These enzymes form a multienzyme metabolic complex, which translocates to the nucleus during S (DNA replication) and G2/M (DNA damage checkpoint) phases, and is required for biosynthesis of the nucleotide precursor, thymidylate, which is critical to DNA synthesis and repair. This mechanism uses 5,10-methyleneTHF, although, alternatively, 5,10-methyleneTHF may also be redistributed towards the methionine pathway. This latter metabolic process involves the folate- and $\mathrm{B}_{12}$-dependent remethylation of homocysteine for genomic and non-genomic methylation, catalysed by the enzyme methylenetetrahydrofolate reductase (MTHFR) through a non-reversible process (Fig. 1). ${ }^{15,16}$ Therefore, a balance exists in how folate is distributed between these two metabolic pathways, with competition for the 5,10-methyleneTHF cofactor existing at the nexus of these pathways.

Similar to the DNA nucleobases, folate vitamers are capable of absorbing UVR and are now established as being photosensitive. The absorption of UV radiation by folates ranges from 280-370 $\mathrm{nm}$, indicating that these compounds absorb a spectrum of wavelengths that include UV-C, UV-B and UV-A radiation. In the presence of UVR, reduced folates may undergo irreversible oxidative cleavage into by-products of pteridine and p-aminobenzoylglutamate, both of which lack vitamin activity. ${ }^{17,18}$ In a biological context, photosensitive folates circulate in the vascular system, along with a number of compounds including the flavins and porphyrins, which are natural photosensitizers. Upon UVR exposure, these compounds react to produce reactive oxygen species (ROS), which have the potential to readily oxidise circulating folates. ${ }^{19-21}$

One of the most UV-sensitive folates is 5-methyltetrahydrofolate (5-methylTHF), produced via the activity of the MTHFR enzyme. ${ }^{14}$ Alongside the vitamer, the MTHFR enzyme itself has also been found to be UV-responsive. In a 2012 study by Yafei et al, epidemiological data showed a significant association between UVR exposure and population frequency of the $\mathrm{T}$ allele of a common genetic variant of the MTHFR gene (MTHFR-C677T). ${ }^{22}$ Interestingly, UV levels have also been shown to influence activity of the cSHMT enzyme, with a preliminary study observing translation of this enzyme as increased significantly in UV-exposed cells. ${ }^{23}$ This finding provides novel evidence to suggest a potential relationship between UV levels and the enhanced activity of the DNA repair multienzyme complex, of which cSHMT is a part, during times of UV-induced DNA damage. ${ }^{16}$

Common gene variants within this multienzyme complex are likely to modulate cellular response to UVR through alteration of the ability of these enzymes to utilize folate and translocate to the nucleus. Candidate polymorphisms in this context include $\mathrm{cSH}$ $M T-C 1420 \mathrm{~T}$, a common gene variant in $c S H M T$ which results in an amino acid substitution that prevents the multienzyme complex from undergoing localization to the nucleus. ${ }^{24}$ Two candidate polymorphisms exist for the TYMS gene, one being the TYMS double or triple 28-base pair tandem repeat (TYMS 28bp 2R $>3 \mathrm{R}$ ) that occurs at the promoter region of the enzyme and the second being the TYMS 6-base pair insertion (TYMS 3'UTR ins/del) variant that occurs in the enzyme region containing binding sites for regulatory proteins and mRNA. ${ }^{25,26}$ Carriage of the $3 \mathrm{R}$ and insertion alleles for these variants results, respectively, in higher translation efficiency and negatively altered mRNA stability. ${ }^{25,26}$ Furthermore, a polymorphism of the DHFR enzyme resulting in a 19bp deletion within the site of a key regulatory sequence has been theorized 
Jones P. et al: UV Responsive Folate Genes

Table 1. Gene frequency information for folate polymorphisms

\begin{tabular}{|c|c|c|c|c|c|c|c|c|}
\hline \multirow{2}{*}{ Polymorphism } & \multirow{2}{*}{ Studies, $n$} & \multirow{2}{*}{$\begin{array}{l}\text { Total } \\
\text { Subjects, } n\end{array}$} & \multicolumn{2}{|c|}{ Minimal Frequency } & \multicolumn{2}{|c|}{ Maximal Frequency } & \multirow{2}{*}{$\begin{array}{l}\text { Collated } \\
\text { Frequency }^{a}\end{array}$} & \multirow{2}{*}{$\begin{array}{l}\text { Hardy- } \\
\text { Weinberg }\end{array}$} \\
\hline & & & Frequency & Latitude & Frequency & Latitude & & \\
\hline SHMT1-C1420T (rs1979277) & 25 & 12,126 & 0.25 & 44.5 & 0.61 & 17.4 & 0.36 & 0.464 \\
\hline MTHFR-C677T (rs1801133) & 41 & 23,570 & 0.08 & 17.4 & 0.47 & 45.5 & 0.34 & 0.772 \\
\hline MTHFR-A1298C (rs1801131) & 19 & 7,029 & 0.09 & 28.6 & 0.40 & 38.4 & 0.31 & 0.628 \\
\hline TYMS-28bp 2R>3R (rs34743033) & 35 & 10,109 & 0.38 & 58.2 & 0.74 & 34.1 & 0.53 & 0.576 \\
\hline $\begin{array}{l}\boldsymbol{T Y M S} \text {-3'UTR ins/del } \\
\text { (rs16430/rs151264360) }\end{array}$ & 11 & 2,357 & 0.25 & 36.72 & 0.56 & 39.9 & 0.39 & 0.655 \\
\hline DHFR 19bp deletion (rs70991108) & 12 & 2,142 & 0.26 & 39.9 & 0.57 & 29.4 & 0.42 & 0.740 \\
\hline
\end{tabular}

${ }^{a}$ Collated frequency was calculated as the average frequency of the polymorphic allele, and was used in Hardy-Weinberg equilibrium testing of each polymorphism.

to exhibit negative effects on the expression and activity of this enzyme. $^{27}$

Competition in the distribution of 5,10-methyleneTHF between thymidylate biosynthesis and remethylation pathways indicates that common MTHFR variants (C677T and A1298C) may also alter activity of the multienzyme complex in thymidylate production during DNA repair. Furthermore, these polymorphisms are associated with decreased MTHFR enzyme activity and hence alter the pool of 5,10-methyleneTHF substrate available for other biosynthetic pathways in the folate system (i.e. thymidylate/methionine biosynthesis). ${ }^{22,28}$

Given the new evidence that indicates key metabolism enzymes of the folate system are capable of responding to UV and UV-induced damage, it has been hypothesized that functionality of the DNA repair complex will reflect UV exposure. It is suspected that, to counteract possible increases in UV-induced DNA damage, a greater requirement for the nuclear localization of this multienzyme complex and the availability of 5,10-methyleneTHF substrate is needed in the case of high UV exposure. The aim of this study was, therefore, to explore the possible association of global UV levels on the prevalence of polymorphisms in key folate metabolism enzymes.

\section{Methods}

\section{Polymorphism data}

A collation of data for the polymorphisms MTHFR-C677T (rs1801133), MTHFR-A1298C (rs1801131), cSHMT-C1420T (rs1979277), TYMS 28bp 2R>3R (rs34743033), TYMS 3'UTR ins/ del (rs16430/rs151264360) and DHFR 19bp deletion (rs70991108) was performed using a computerized search of the PubMed database. All searches took place before 10/04/2016 using the key terms of 'serinehydroxymethyl transferase', 'methylenetetrahydrofolate reductase', 'thymidylate synthase' and 'dihydrofolate reductase' or their abbreviated forms (SHMT, MTHFR, TYMS/TS, DHFR) along with their respective gene variants: MTHFR-C677T and A1298C, cSHMT-C1420T, TYMS 28bp 2R >3R and 3'UTR ins/ del and DHFR-16bp deletion.

Of the returned results, studies containing a healthy cohort or control groups consisting of 40 participants or more as part of their study design were included for analysis. Included studies had been undertaken in Africa and the Eurasia region, including all of Europe and the southwestern parts of Asia. Studies from the African and Eurasia region were chosen in accordance with them being well defined, and containing populations with relatively limited admixture; each population was predominately archaic in respect to skin pigmentation and other factors likely to influence the degree of cellular UV damage. Human's second "out-of-Africa" migration encompasses these global areas, with this divergence giving rise to Caucasian/European/Middle Eastern/south Asian ancestral components, which was followed by a migration back to Northern Africa. ${ }^{29}$ From reported studies found to fit the outlined selection criteria, genotype frequencies of control groups or healthy cohorts were recorded. Location of each study was documented.

\section{Global UVR levels}

The African and Eurasian global regions cover a wide latitudinal range, with geographic latitude being a static surrogate measure of global UV-levels. Geographic latitude is a routinely used measure for UVR exposure; this applicability is supported by the finding of Cahoon et al demonstrating that latitude of residence explains the greatest proportion of variability in daily individual UV exposure. ${ }^{30}$ The latitudinal location of each study was used as a surrogate measure of population UV exposure in the present study. Latitude coordinates provided online (http://www.findlatitudeandlongitude.com/) were used.

\section{Statistical analysis}

Genotype data from selected studies were used to calculate allele frequency in the control or healthy cohort study groups. Allele frequency was tested against the corresponding study's latitudinal location. Statistical analysis was performed using JMP (version 11; SAS Institute Inc., Cary, NC, USA). Association between allele frequencies and the study latitudinal position was examined using a regression analysis. Hardy-Weinberg equilibrium was tested for each polymorphism using the $\chi^{2}$ test.

\section{Results}

According to the search results and defined exclusion criteria, a total of 51 studies were found suitable for analysis (Supplementary information $\mathrm{S} 1$ ). These selected studies, combined, represented 28,678 healthy individuals living in Africa and the Eurasia area. Frequency of polymorphisms did not deviate from Hardy-Weinberg equilibrium; information on frequency data for each variant is outlined in Table 1. 


\section{Relationship between frequency of MTHFR polymorphisms and latitude}

Frequency of the MTHFR-C677T T allele in the Eurasia region is shown in Figure 2. Frequency of the polymorphic $\mathrm{T}$ allele was found to be significantly positively associated with latitude, with an increase in degrees of latitude being associated with an increase in polymorphism frequency $\left(r^{2}=0.14, p=0.017\right)$. Similarly, a positive latitude-dependent relationship was seen for the polymorphic $\mathrm{C}$ allele of the MTHFR-A1298C variant $\left(r^{2}=0.37, p=0.005\right)$ (Fig. 2$)$.

\section{Relationship between frequency of SHMT-C1420T and latitude}

The $\mathrm{T}$ allele frequency of the cSHMT-C1420T polymorphism across Eurasia is shown in Figure 2. A significant negative latitude-dependent relationship was detected for carriage of the $\mathrm{T}$ allele, with occurrence of the $c S H M T-C 1420 T$ polymorphic allele decreasing with increase in latitude $\left(r^{2}=0.73, p<0.001\right)$.

\section{Relationship between frequency of TYMS/DHFR polymorphism and latitude}

Analysis of TYMS 28bp 2R $>3 \mathrm{R}$ frequency in reported African and Eurasia studies found a significant negative relationship between latitude and frequency of the $3 \mathrm{R}$ allele $\left(r^{2}=0.12, p=0.039\right)$ (Fig. 2). A negative trend was observed for the frequency of the TYMS 6bp ins/del gene variant across Africa and Eurasia, but this was not significant $\left(r^{2}=0.28, p=0.096\right)$. Additionally, no significant relationship between latitude and DHFR 19bp deletion frequency was found $\left(r^{2}=0.05, p=0.496\right)$ (Fig. 2).

\section{Discussion}

The findings presented herein indicate an association between UV exposure and the activity of enzymes cSHMT, TYMS and MTHFR, with results supporting those documented for the distribution of the MTHFR-C677T variant. ${ }^{22}$ Interaction between genes, termed epistasis, is a phenomenon increasingly highlighted in the area of genetic epidemiology. The results of this study suggest the existence of epistatic actions between folate metabolism enzymes within two key metabolic pathways: those associated with DNA synthesis and repair, as well as remethylation processes. In reviewing these findings in concert with those of previous studies, plausible biological and evolutionary models may be proposed for the association of UVR on the folate system.

Given the importance of both methylation and de novo thymidylate biosynthesis processes and their obligate need to utilize 5,10-methyleneTHF, a strong rationale exists to examine genetic variation in key enzymes involved in both pathways in the context of the UVR influence on folate metabolism. This study documents a positive association between frequency of MTHFR-C677T and MTHFR-A1298C polymorphisms and latitude, with increases in latitude significantly associated with increased frequency of the polymorphic allele of these variants. The MTHFR polymorphisms are associated with an up to $70 \%$ decline in enzymatic activity, impairing methylation processes in the cell and resulting in elevations in homocysteine. ${ }^{31}$ These variants are additionally proposed to result in a more robust de novo thymidylate biosynthesis as a consequence of declining amounts of 5,10-methyleneTHF being available for irreversible reduction to 5 -methylTHF via ac- tion of the MTHFR enzyme. This event increases the amount of 5,10-methyleneTHF available to be channelled into de novo thymidylate synthesis. ${ }^{32}$ The positive association between frequency of MTHFR-C677T and MTHFR-A1298C polymorphisms and latitude documented in the current study suggests that in areas closer to the equator, which are increasingly exposed to UVR, activity of the MTHFR enzyme reflects greater functional competency because of the absence of the MTHFR polymorphisms. This begs the question as to whether there may be a tendency for MTHFR activity, and hence methylation and thymidylate pathways to be modulated by a changing UV environment.

The current study documented a negative association between frequency of both the TYMS 28bp 2R $>3 \mathrm{R}$ and $c S H M T-\mathrm{C} 1420 \mathrm{~T}$ variants and latitude, with increases in latitude significantly associated with a decline in frequency of these polymorphisms. The folate-mediated enzymes TYMS and cSHMT, alongside DHFR, are components within a nuclear multi-enzyme complex located at the replication fork during DNA replication and repair. The complex is responsible for synthesis of the DNA precursor, thymidylate, working alongside a group of 'replitase' enzymes (DNA polymerase, ribonucleotide reductase, thymidine kinase and NDP kinase) which act as the DNA replication and repair apparatus. ${ }^{33}$ Impaired activity of the folate-dependent enzyme complex in this system results in uracil being misincorporated into DNA in the place of thymine, with subsequent DNA instability. ${ }^{33}$

Activity of the thymidylate synthase enzyme is the rate limiting step in this complex, responsible for synthesizing the thymine precursor, thymidylate, during DNA repair. ${ }^{25}$ Two functional polymorphisms associated with expression of this enzyme were explored in this study, with the TYMS $28 \mathrm{bp} 2 \mathrm{R}>3 \mathrm{R}$ variant found to be significantly associated with latitude. The $3 R$ allele for this variant is associated with a 2-4 fold increase in expression of the TYMS enzyme, as a result of the effect of this variant on the translation efficiency of TYMS. ${ }^{34}$ Consequently, turnover of 5,10-methyleneTHF via the TYMS enzyme is proposed to be greater in 3R3R individuals, with a decrease in this vitamer and an increase in homocysteine levels previously observed in such individuals. ${ }^{35,36} \mathrm{Ob}-$ served elevations in homocysteine in 3R3R individuals imply the methylation pathway is being hindered in favour of de novo thymidylate synthesis. The negative association between frequency of the TYMS 28bp 2R $>3 \mathrm{R}$ and latitude recorded in the current study suggests that in geographic areas exposed to increasing levels of UVR, activity of the TYMS may be enhanced via the presence of this variant, possibly at the expense of methylation processes.

The association between TYMS 28bp 2R $>3 \mathrm{R}$ and latitude suggests a potential trend towards de novo thymidylate biosynthesis being enhanced in geographic areas increasingly exposed to UVR, as a likely mechanism to counteract increases in UV-induced DNA damage. However, this is challenged by the negative association between frequency of the $c S H M T-\mathrm{C} 1420 \mathrm{~T}$ variant and latitude documented in this study. This association implies that in locations exposed to greater UVR, the nuclear localization of the multienzyme complex is increasingly inhibited via the cSHMT-C1420T polymorphism.

In humans, the SHMT enzyme exists in two isoforms, with the cSHMT gene encoding the cytosolic/nuclear enzyme (cSHMT) and the $M S H M T$ gene encoding a mitochondrial SHMT enzyme. These isoforms have distinct cellular roles but possess high sequence identity $(66 \%)$ and comparable enzyme properties. ${ }^{37} \mathrm{~A}$ preliminary study has reported that the mSHMT enzyme allows for functional redundancy in cases where activity of the cSHMT enzyme is inhibited, and is up-regulated in cells lacking cSHMT during DNA replication and repair cell phases. ${ }^{24}$ With respect to this, the negative association between frequency of the $c S H M T$ - 
A
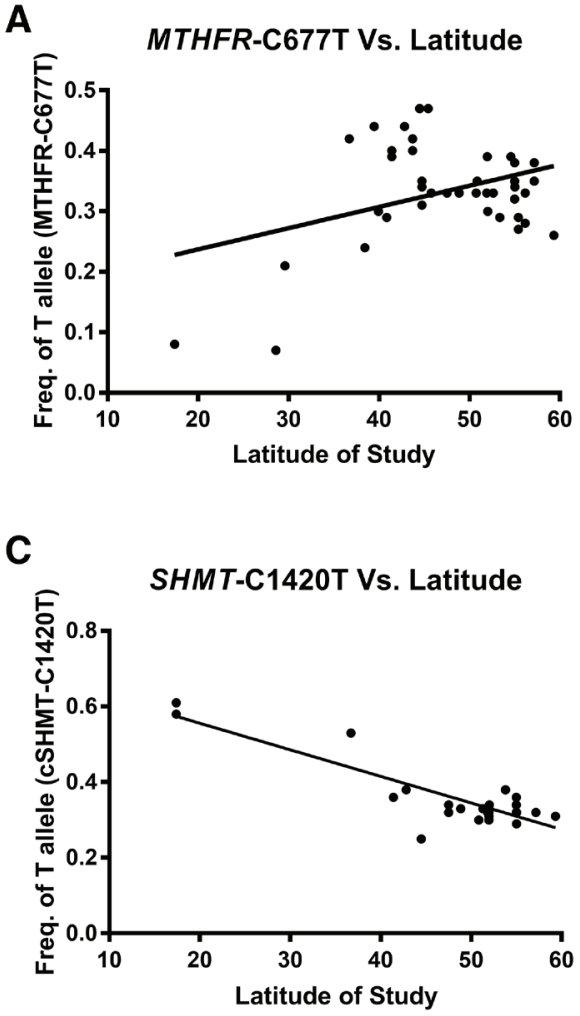

E

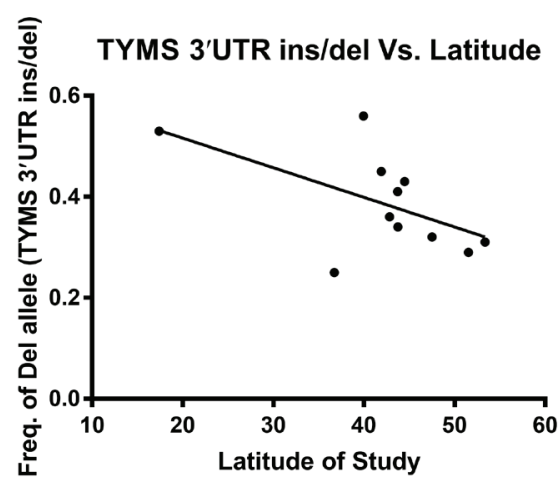

B

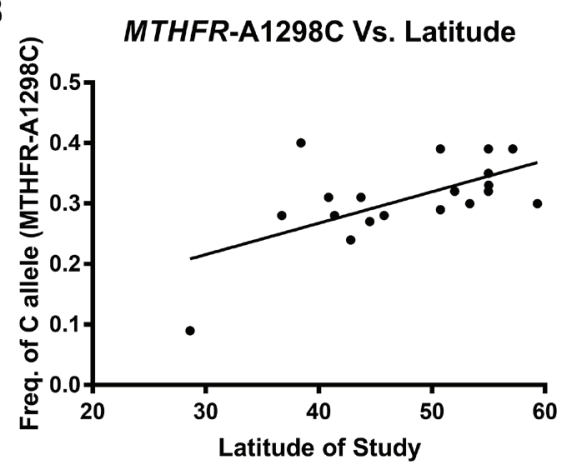

D

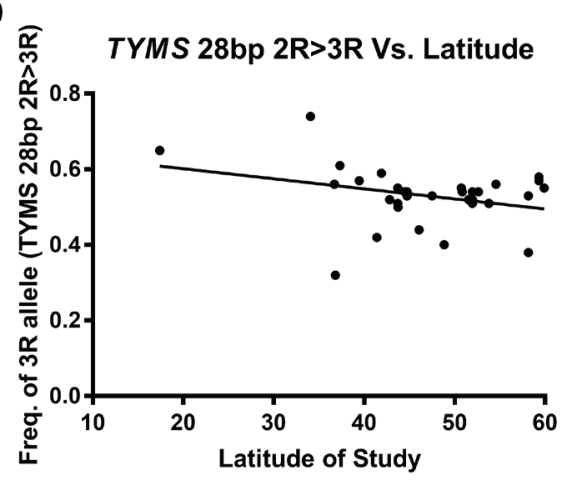

$\mathbf{F}$

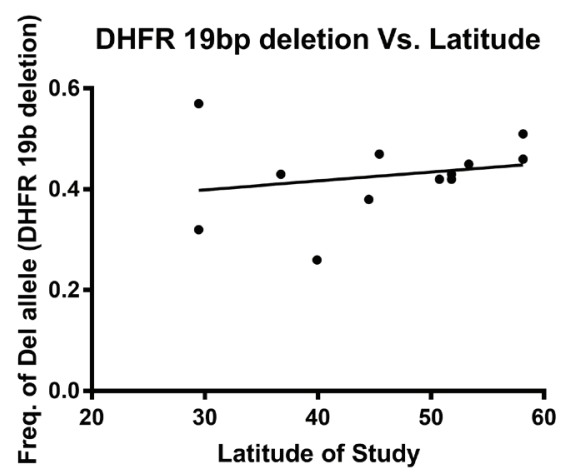

Fig. 2. Scatterplots showing the association between frequency of a) $M T H F R-C 677 \mathrm{~T}$, b) $M T H F R-A 1298 \mathrm{C}$, c) $c S H M T-\mathrm{C} 1420 \mathrm{~T}$, d) $T Y M S$ 28bp $2 \mathrm{R}>3 \mathrm{R}$ e) $T Y M S$ 3'UTR ins/ del and f) $D H F R$ 19bp deletion polymorphic alleles according to latitude.

C1420T variant and latitude documented in this study may indicate that activity of the de novo thymidylate synthesis pathway is increasingly impaired in populations exposed to higher UVR, but the burden of this variant is unclear due to mSHMT likely maintaining cSHMT activity in such cases. However, analysis of $c S H M T$ and $m S H M T$ gene sequences finds the $m S H M T$ gene likely originally arose from the $c S H M T$ gene. ${ }^{38}$ This proposes an interesting question; does the existence of two SHMT isoforms in the body serve to support activity of thymidylate biosynthesis in varying UV environments?

Associations between the frequency of MTHFR-C677T, MTHFR-A1298C, TYMS 28bp 2R $>3 \mathrm{R}$ and cSHMT-C1420T polymorphisms and latitude indicate a distinct but differential clinal response to UVR. Each association suggests an independent mech- anism by which the de novo thymidylate synthesis and remethylation pathways may be influenced by UV environment. However, in considering all associations en masse, the findings do not appear to point to a tendency of folate genotype to be selected in a manner that favours specific pathways in folate metabolism in particular UV environments, as hypothesized. Instead, these findings provide novel evidence to suggest folate genotypes are selected to maintain homeostasis between de novo thymidylate synthesis and methylation processes at differing UVR levels. This is supported by associations between latitude and both MTHFR polymorphisms, as well TYMS 28bp 2R $>3 \mathrm{R}$, with these variants associated with elevations in homocysteine levels in previous studies. Preliminary studies indicate that in cases where these polymorphisms occur together, an exacerbated build-up of homocysteine in the cell may occur. Popu- 
lation frequency of both MTHFR variants and TYMS $28 \mathrm{bp} 2 \mathrm{R}>3 \mathrm{R}$ were demonstrated to have reciprocal frequencies, suggesting that frequency of these polymorphisms occur in a manner that limits the incidence of their combined effect on the methylation pathway, permitting activity of the methylation pathway to be sustained in differing UV environments.

There are limitations to the current study; the number of studies providing sufficient data for frequency of the TYMS 3'UTR ins/del and DHFR 19bp deletion polymorphisms was relatively low. Further study power is required to assess if there is a relationship between these gene variants and UVR. Furthermore, studies reviewing the relationship between UVR and human biology are hindered by the infeasibility of measuring extended UVR exposure of populations. Static ecological variables, such as latitude, are therefore commonly used as surrogate measures of UVR exposure. The use of latitude as a UVR measure is based on the relationship of latitude with solar zenith angle, with this angle smallest at the equator and increasing as latitude increases towards the poles. Increases in the solar zenith angle reflect an increase in the path sunlight travels through the atmosphere, where the amount of UVR reaching the Earth's surface is reduced through scattering and absorption. ${ }^{39} \mathrm{~A}$ further limitation of the current study is that levels of UVR may be influenced by a number of additional geographical and meteorological factors, such as cloud cover, surface reflectance and altitude. ${ }^{40}$ However, as outlined, latitude explains the greatest proportion of variability in UV exposure over other such measures, ${ }^{30}$ with the correlation between latitude and UVR levels well represented by satellite data (Fig. S1). Consequently, a number of preliminary studies have used study latitude as a confident measure of UVR exposure; however, additional indices of UV exposure, such as UV index, have also been used. ${ }^{41-44}$ Future studies may benefit from utilizing a number of measures of UV exposure, whilst considering additional factors that may influence UV radiation levels, to further elucidate the role of UVR on folate metabolism.

It was initially hypothesized that DNA repair would be enhanced in high UVR locations, through the increased activity of the thymidylate synthesis pathway. This was examined by assessing associations between frequency of polymorphisms in key metabolism enzyme genes and latitude, with findings unsupportive of this hypothesis but suggesting that folate genotypes are selected to maintain homeostasis within the folate system. Given the consequences of genotoxic stress, counteracting DNA damage in the body may take the form of firstly, DNA repair mechanisms being enhanced, or secondly, preventing DNA damage through strengthening the integrity of the epidermal barrier between UVR and our UV-labile molecular components. Whilst study findings seem to not support folate genotype being selected to enhance DNA repair pathways in high UVR environments, the relationship between UVR and folate is proposed to have a role in strengthening our epidermal barrier.

The range of skin pigmentation observed in humans is an apparent adaptation to UVR levels differing by latitude. ${ }^{45}$ The vitamin D-folate hypothesis highlights a potential role for folate in preventing DNA damage through driving the evolution of skin pigmentation. This hypothesis proposes the cline in skin pigmentation is an adaptive response to UVR and the need to protect vitamins that are key to ensuring reproductive success. This hypothesis suggests the evolution of dark skin protected against UV loss of folate, and the occurrence of lighter skin tones in areas of low UVR allowed for adequate vitamin D synthesis. ${ }^{45}$ The present findings suggest a potential homeostasis in the folate system that is maintained in differing UVR environments via folate gene epistasis. This is supported by the evidence that human skin pigmentation has evolved in parallel to our UVR environment, as a likely mechanism to maintain a balanced biological influence of UVR, irrespective of latitude. The presented findings are not inconsistent with the vitamin D-folate hypothesis, but many other mechanisms have been reported as factors that drive the evolution of our skin phenotype. ${ }^{45}$

In showing significant associations between latitude as a surrogate measure of UVR and the frequency of four gene polymorphisms that influence folate cofactor partitioning in folate metabolism, the present findings may provide new insight into folate-related diseases. Since the 1990s, folate status and genetics have been linked to a plethora of diseases, many of which stem from impaired DNA-thymidylate and methylation pathways, and include adverse prenatal, oncogenic, cardiovascular and neurological outcomes. ${ }^{46}$ Folate status and genotype are independent but interacting risk factors in these outcomes, with occurrence of a number of these diseases also showing seasonal variability and reflecting changes in global UVR levels. ${ }^{47-49}$ Given that the epistatic interactions presented here are key to folate-related thymidylate and methylation pathways, it seems likely that environmental factors such as UVR will prove to be an important determinant of disease occurrence in future studies.

The folate-dependent gene variants MTHFR-C677T, MTHFRA1298C, TYMS 28bp 2R $>3 \mathrm{R}$ and cSHMT-C1420T exhibit a differential global occurrence in response to UVR. Frequency of the variant allele decreases towards the equator for the two MTHFR variants, but increases for the TYMS and SHMT variants. Based on these findings, the authors hypothesize that a potential homeostasis exists in the folate system that is maintained in differing UVR environments via folate-related gene epistasis. Such a mechanism would be driven by the UVR sensitivity of folate. Adaptation in the form of optimal folate-related gene epistasis and skin pigmentation would be selected to maintain provision of folate coenzymes for crucial methylation and DNA-thymidylate biosynthetic processes. Future research is required to examine the extent to which folaterelated epistatic interactions respond to environmental cues, such as UVR exposure.

Future exploratory work should examine whether this "clinal" response in folate homeostasis is an adaptive component within current models of the evolution of skin pigmentation (the vitamin D-folate hypothesis). Furthermore, the relevance of this environment-diet-gene interaction to the patho-aetiology of contemporary disease needs to be explored with a considered approach, taking into account interactions between skin pigmentation, UVR exposure, folate status and folate-related gene epistasis. An improved understanding of the relationship between the folate system, UVR environment, and folate-related health outcomes may provide new insight into humankind's continued vulnerability to these diseases. This study provides the first evidence of an association between latitude and the occurrence of MTHFR-A1298C, TYMS $28 \mathrm{bp} 2 \mathrm{R}>3 \mathrm{R}$ and $c S H M T-\mathrm{C} 1420 \mathrm{~T}$ polymorphisms, and is first to introduce the idea of UVR environments influencing folate-related gene epistasis; with this having far reaching consequences for both evolutionary constructs and mechanisms of disease.

\section{Conclusion}

This study documented associations between frequency of $M T H-$ FR-C677T, MTHFR-A1298C, TYMS 28bp 2R $>3 \mathrm{R}$ and $c S H M T$ C1420T polymorphisms and latitude as a surrogate measure for UVR. Frequency of the MTHFR-C677T and MTHFR-A1298C polymorphisms were found to be positively associated with latitude, with a negative association between latitude and frequency of the cSHMT-C1420T and TYMS 28bp 2R>3R variants also ob- 
served. These findings provide novel evidence to suggest that folate genotype is selected to maintain homeostasis between folate de novo thymidylate synthesis and methylation pathways in environments of differing UV levels. To the authors' knowledge, this is the first study to report significant associations between latitude and the occurrence of MTHFR-A1298C, TYMS 28bp 2R $>3 \mathrm{R}$, and cSHMT-C1420T polymorphisms. On-going studies are required to further explore the biological significance of these findings, and the relevance of these findings to human phenotype and disease.

\section{Conflict of interest}

The authors have no conflict of interests related to this publication.

\section{Author contributions}

Analysis \& interpretation of data (PJ, EB, ML), statistical analysis (PJ, EB, ML), manuscript writing (PJ, ML), critical revision (EB, $\mathrm{ZY}, \mathrm{MV}, \mathrm{ML})$, material support (MV).

\section{Supplementary information}

(Supplementary information is linked to the online version of the paper on the Exploratory Research and Hypothesis in Medicine website.)

\section{References}

[1] Nesse RM. How is Darwinian medicine useful? West J Med 2001;174:358-360.

[2] Dobzhansky T. Nothing in biology makes sense except in the light of evolution. Am Biol Teach 1973;35:125-129.

[3] Richa, Sinha RP, Hader DP. Physiological aspects of UV-excitation of DNA. Top Curr Chem 2015;356:203-248. doi:10.1007/128_2014_531.

[4] Jackson SP, Bartek J. The DNA-damage response in human biology and disease. Nature 2009;461:1071-1078. doi:10.1038/nature08467.

[5] Kastan MB. DNA damage responses: mechanisms and roles in human disease: 2007 G.H.A. clowes memorial award lecture. Mol Cancer Res 2008;6:517-524. doi:10.1158/1541-7786.MCR-08-0020.

[6] Lengauer C, Kinzler KW, Vogelstein B. Genetic instabilities in human cancers. Nature 1998;396:643-649. doi:10.1038/25292.

[7] Kulkarni A, Wilson DM 3rd. The involvement of DNA-damage and -repair defects in neurological dysfunction. Am J Hum Genet 2008;82:539-566. doi:10.1016/j.ajhg.2008.01.009.

[8] Rass U, Ahel I, West SC. Defective DNA repair and neurodegenerative disease. Cell 2007;130:991-1004. doi:10.1016/j.cell.2007.08.043.

[9] Matzuk MM, Lamb DJ. The biology of infertility: research advances and clinical challenges. Nat Med 2008;14:1197-1213. doi:10.1038/nm.f.1895.

[10] Schumacher B, Garinis GA, Hoeijmakers JH. Age to survive: DNA damage and aging. Trends Genet 2008;24:77-85. doi:10.1016/j.tig.2007.11.004.

[11] Mercer J, Mahmoudi M, Bennett M. DNA damage, p53, apoptosis and vascular disease. Mutat Res 2007;621:75-86. doi:10.1016/j.mrfmmm.2007.02.011.

[12] Williams JD, Jacobson EL, Kim H, Kim M, Jacobson MK. Folate in skin cancer prevention. Subcell Biochem 2012;56:181-197. doi:10.1007/978-94-0072199-9_10.

[13] Piskac-Collier AL, Monroy C, Lopez MS, Cortes A, Etzel CJ, Greisinger AJ, et al. Variants in folate pathway genes as modulators of genetic instability and lung cancer risk. Genes Chromosomes Cancer 2011;50:1-12. doi:10.1002/ gcc. 20826 .

[14] Lucock M. Folic acid: nutritional biochemistry, molecular biology, and role in disease processes. Mol Genet Metab 2000;71:121-38. doi:10.1006 mgme.2000.3027.

[15] Stover PJ, Field MS. Trafficking of intracellular folates. Adv Nutr 2011;2:325331. doi:10.3945/an.111.000596.

[16] Anderson DD, Woeller CF, Chiang EP, Shane B, Stover PJ. Serine hydroxymethyltransferase anchors de novo thymidylate synthesis pathway to nuclear lamina for DNA synthesis. J Biol Chem 2012;287:7051-7062. doi:10.1074/jbc.
M111.333120.

[17] Bailey LB, Gregory JF. Folate Metabolism and Requirements. J Nutr 1999;129:779-782.

[18] Shane B. Folate chemistry and metabolism. In: Bailey LB, ed. Folate in Health and Diseaser. 2nd ed. Boca Raton: CRC Press, 2010:1-24.

[19] Steindal AH, Tam TT, Lu XY, Juzeniene A, Moan J. 5-Methyltetrahydrofolate is photosensitive in the presence of riboflavin. Photochem Photobiol Sci 2008;7:814-818. doi:10.1039/b718907a.

[20] Tam TT, Juzeniene A, Steindal AH, Iani V, Moan J. Photodegradation of 5 -methyltetrahydrofolate in the presence of uroporphyrin. J Photochem Photobiol B 2009;94:201-204. doi:10.1016/j.jphotobiol.2008.12.003.

[21] Juzeniene A, Thu Tam TT, Iani V, Moan J. 5-Methyltetrahydrofolate can be photodegraded by endogenous photosensitizers. Free Radic Biol Med 2009;47:1199-1204. doi:10.1016/j.freeradbiomed.2009.07.030.

[22] Yafei W, Lijun P, Jinfeng W, Xiaoying Z. Is the prevalence of MTHFR C677T polymorphism associated with ultraviolet radiation in Eurasia? J Hum Genet 2012;57:780-786. doi:10.1038/jhg.2012.113.

[23] Fox JT, Shin WK, Caudill MA, Stover PJ. A UV-responsive internal ribosome entry site enhances serine hydroxymethyltransferase 1 expression for DNA damage repair. J Biol Chem 2009;284:31097-31108. doi:10.1074/jbc. M109.015800.

[24] Anderson DD, Stover PJ. SHMT1 and SHMT2 are functionally redundant in nuclear de novo thymidylate biosynthesis. PLoS One 2009;4:e5839. doi:10.1371 journal.pone.0005839

[25] Kealey C, Brown KS, Woodside JV, Young I, Murray L, Boreham CA, et al. A common insertion/deletion polymorphism of the thymidylate synthase (TYMS) gene is a determinant of red blood cell folate and homocysteine concentrations. Hum Genet 2005;116:347-353. doi:10.1007/s00439-004-1243-2.

[26] Horie N, Aiba H, Oguro K, Hojo H, Takeishi K. Functional Analysis and DNA Polymorphism of the Tandemly Repeated Sequences in the 5'-terminal Regulatory Region of the Human Gene for Thymidylate Synthase. Cell Struct Funct 1995;20:191-197.

[27] Johnson WG, Stenroos ES, Spychala JR, Chatkupt S, Ming SX, Buyske S. New 19 bp deletion polymorphism in intron-1 of dihydrofolate reductase (DHFR): a risk factor for spina bifida acting in mothers during pregnancy? Am J Med Genet A 2004;124A:339-345. doi:10.1002/ajmg.a.20505.

[28] van der Put NM, Gabreëls F, Stevens EM, Smeitink JA, Trijbels FJ, Eskes TK, et al. a second common mutation in the methylenetetrahydrofolate reductase gene: an additional risk factor for neural-tube defects? Am J Hum Genet 1998;62:1044-1051. doi:10.1086/301825.

[29] Shriner D, Tekola-Ayele F, Adeyemo A, Rotimi CN. Genome-wide genotype and sequence-based reconstruction of the 140,000 year history of modern human ancestry. Sci Rep 2014;4:6055. doi:10.1038/srep06055.

[30] Cahoon EK, Wheeler DC, Kimlin MG, Kwok RK, Alexander BH, Little MP, et al. Individual, environmental, and meteorological predictors of daily personal ultraviolet radiation exposure measurements in a United States cohort study. PLoS One 2013;8:e54983. doi:10.1371/journal.pone.0054983.

[31] Ma J, Stampfer MJ, Giovannucci E, Artigas C, Hunter DJ, Fuchs C, et al. Methylenetetrahydrofolate reductase polymorphism, dietary interactions, and risk of colorectal cancer. Cancer Res 1997:57:1098-1102.

[32] Rosenblatt DS, Cooper BA, Pottier A, Lue-Shing H, Matiaszuk N, Grauer K Altered vitamin B12 metabolism in fibroblasts from a patient with megaloblastic anemia and homocystinuria due to a new defect in methionine biosynthesis. J Clin Invest 1984;74:2149-2156. doi:10.1172/JCI111641.

[33] Prem veer Reddy G, Pardee AB. Multienzyme complex for metabolic channeling in mammalian DNA replication. Proc Natl Acad Sci U S A 1980;77:3312-3316.

[34] Kawakami K, Omura K, Kanehira E, Watanabe Y. Polymorphic tandem repeat in the thymidylate synthase gene is associated with its protein expression in human gastrointestinal cancers. Anticancer Res 1999;19:3249-3252.

[35] Motti C, Gnasso A, Bernardini S, Massoud R, Pastore A, Rampa P, et al. Common mutation in methylenetetrahydrofolate reductase. Correlation with homocysteine and other risk factors for vascular disease. Atherosclerosis 1998;139:377-383. doi:10.1016/S0021-9150(98)00079-3.

[36] Trinh BN, Ong CN, Coetzee GA, Yu MC, Laird PW. Thymidylate synthase: a novel genetic determinant of plasma homocysteine and folate levels. Hum Genet 2002;111:299-302. doi:10.1007/s00439-002-0779-2.

[37] Giardina G, Brunotti P, Fiascarelli A, Cicalini A, Costa MG, Buckle AM, et al. How pyridoxal 5 -phosphate differentially regulates human cytosolic and mitochondrial serine hydroxymethyltransferase oligomeric state. FEBS 2015;282:1225-1241. doi:10.1111/febs.13211.

[38] Garrow TA, Brenner AA, Whitehead VM, Chen XN, Duncan RG, Korenberg $\mathrm{JR}$, et al. Cloning of human cDNAs encoding mitochondrial and cytosolic serine hydroxymethyltransferases and chromosomal localization. J Biol Chem 1993;268:11910-11916.

[39] Bilinski K, Byth K, Boyages J. Association between latitude and breast cancer incidence in mainland australian women. J Cancer Res 2014;2014:1-9. doi: $10.1155 / 2014 / 149865$.

[40] D'Orazio J, Jarrett S, Amaro-Ortiz A, Scott T. UV radiation and the skin. Int J Mol Sci 2013;14:12222-12248. doi:10.3390/ijms140612222.

[41] Grant WB. Does solar ultraviolet irradiation affect cancer mortality rates in 
China? Asian Pac J Cancer Prev 2007;8:236-242.

[42] Yu GP, Hu DN, McCormick SA. Latitude and incidence of ocular melanoma Photochem Photobiol 2006;82:1621-1626. doi:10.1562/2006-07-17-RA-970.

[43] Handel AE, Jarvis L, McLaughlin R, Fries A, Ebers GC, Ramagopalan SV. The epidemiology of multiple sclerosis in scotland: inferences from hospital admissions. PLoS One 2011;6(1):e14606. doi:10.1371/journal.pone.0014606.

[44] Grant WB. An ecologic study of dietary and solar ultraviolet-B links to breast carcinoma mortality rates. Cancer 2002;94:272-281. doi:10.1002/cncr.10196.

[45] Jablonski NG. The evolution of human skin and skin color. Annu Rev Anthropol 2004;33:585-623. doi:10.1146/annurev.anthro.33.070203.143955.

[46] Stanger O. Physiology of folic acid in health and disease. Curr Drug Metabo- lism 2002;3:211-223. doi:10.2174/1389200024605163.

[47] Lim HS, Roychoudhuri R, Peto J, Schwartz G, Baade P, Møller H. Cancer survival is dependent on season of diagnosis and sunlight exposure. Int J Cancer 2006;119:1530-1536. doi:10.1002/ijc.22052.

[48] Marzullo G, Fraser FC. Similar rhythms of seasonal conceptions in neural tube defects and schizophrenia: a hypothesis of oxidant stress and the photoperiod. Birth Defects Res A Clin Mol Teratol 2005;73:1-5. doi:10.1002/bdra.20100.

[49] Marti-Soler H, Gonseth S, Gubelmann C, Stringhini S, Bovet P, Chen PC, et al. Seasonal variation of overall and cardiovascular mortality: a study in 19 countries from different geographic locations. PLoS One 2014;9:e113500. doi:10.1371/journal.pone. 0113500 . 